\title{
The Theory of Unitrons - A Classical Physical Approach for Fundamental Particles and Forces
}

\author{
Tibor Forster \\ Retired from the Institute of Isotopes, Budapest, Hungary. \\ Correspondence: forster.t51@gmail.com
}

\begin{abstract}
This fundamentally new conception is a candidate for the Theory of Everything. According to this classical physical approach, all elementary particles are electric multipole composite and made up of smaller, fundamental building blocks named unitrons. The unitron is the ultimate constituents of matter with an invariable mass, spatial extension and positive $(\mathrm{u}+)$ or negative $(\mathrm{u}-)$ electric charge. There is only one fundamental interaction between unitrons: the attractive or repulsive electric force, which corresponds to the macroscopically described Coulomb force. The model can explain the electric, magnetic, gravitational and nuclear forces furthermore the inertia and inertia forces as the manifestation of the attractive and repulsive fundamental forces of unitrons. The fundamental force propagates at speed of light. It holds the elementary particles together and determines their motion too. Every elementary particle contains both positively and negatively charged unitrons; their net charge is a resultant charge of unitrons. The elementary particles can be described as electromagnetic gyroscopes. The electromagnetic radiation is a wave-like change in the electromagnetic field generated by the electromagnetic force of a photon spinning around its axis. The energy of a photon is proportional to its rotational frequency. Only this rotational frequency changes when it is absorbed or emitted.
\end{abstract}

Keywords: theory of everything; elementary particles; structure of atomic nuclei; fundamental interactions; magnetism; gravity; electromagnetic radiation; inertia; redshift; cosmic background radiation

\section{Introduction}

Although the Standard Model [1] is believed to be theoretically self-consistent, a Theory of Everything (TOE) that fully explains and links together all physical aspects of the Universe is one of the major unsolved problems in physics. Achieving TOE by the unification of the fundamental interactions [2] is believed impossible because the realm of elementary particles might be not representing the deepest level of the Universe. Theory of Unitron, in explicated form, the Theory of Fundamental Forces and Particles (TFPF), as a novel candidate for TOE, suggests reconsideration the perpetual question: What is the Universe, at a fundamental level, truly made out of? According to TFPF, elementary particles are real particles even if some experiments show them waves or point-like entities. All of the elementary particles have definite mass and spatial extensions and most of all they are made up of smaller, electrically charged particles called unitrons. Unitrons are considered the ultimate constituents of matter. The assumption of the existence of the realm of unitrons is not a speculative hypothesis but a clear deduction from the classical physical interpretation of the annihilation radiation. We can describe every elementary particle and their interactions if elementary particles are considered different compositions of electrically charged sub-elementary, i.e. fundamental particles called unitrons. (The term "fundamental" will be used as an equivalent for sub-elementary; the term "elementary" will be used conventionally, e.g. for photon, electron, etc.) Accordingly, the matter is not other than different compositions of charged unitrons that have the very same and invariable mass, spatial extension, and electric charge. The unitrons imagining as rigid spheres exist in two forms; the number of positively $\left(\mathrm{u}^{+}\right)$and negatively $\left(\mathrm{u}^{-}\right)$charged unitrons is the very same. It is hypothesized that every unitron generates sphere symmetric electric field around itself continuously that is propagated at the speed of light. The unitrons not only generate but also accept electric force generated by all other unitrons. The magnitude and direction of the electric force generated by unitrons can be calculated by Coulomb's law. The electric force is no other but an exertion on unitrons to move them accelerated towards the source unitron or to the opposite direction. According to the TFPF, the Universe on the ultimate level is not other than the totality of unitrons in dynamic equilibrium in the infinite space and time. The position and movement of fundamental particles in space and time are determined by the forces generated the electrically charged fundamental particles themselves. The organization of substance on the fundamental level is 
as simple and clear as classical physics becomes applicable for the description of the microscopic world. According to the TFPF, the laws of conservation of mass and electric charge, the notion of absolute space and time, Newton's laws, Coulomb's law, Galilean relativity, and other classical physical laws are valid also in microscopic level. The energy is an important property of matter but it is not equivalent to the mass. The classical theories were supplemented by only one non-restrictive postulate, namely, that the propagation speed of force is $c=299,792,458 \mathrm{~m} / \mathrm{s}$. Due to the classical physical description, this alternative explanation of elementary particles and their interaction is comprehensible and conforms to the macroscopic world. Due to their electric charge, unitrons can never occur in single form thus we shall have never got direct evidence of their existence; only the consistency of the theory can justify the existence of unitrons. Basics of TFPF are described mainly qualitatively, as the fast sharing of an alternative model seemed more reasonable than its single-handed elaboration.

\section{Fundamental Particles - Elementary Particles}

According to modern physics [3], annihilation radiation and pair production are demonstrative examples of matter transforming into energy:

$$
\mathrm{e}^{+}+\mathrm{e}^{-} \rightleftarrows 2 \gamma^{0}(511 \mathrm{keV})
$$

Using a classical physical approach and bearing in mind the law of conservation of mass and electric charge, these processes can and must be interpreted completely differently. It is assumed that during "annihilation radiation" the positron and the electron stick together to form an unstable intermediate, which immediately decays releasing two identical photons of high energy. Thus, two elementary particles composed of matter transform into two other elementary particles which are also composed of matter. The reverse of the process may also occur in the form of pair production.

Consequently, the electron, the positron and the photon are not indivisible "elementary" particles; instead, they are composites. According to the law of conservation of mass, the mass of the two identical photons is equal to that of the electron and the positron. Both the electron and the positron, and as a result, the photon is made up of more than one electrically charged particle. The photon inherits half of the charge of the electron and half of the charge of the positron, which makes its net charge zero. Since two identical photons are created, it can be assumed that the negative electric charge of the electron is carried at least by two particles. It is assumed, an attraction of oppositely charged particles holds together the negative repulsive particles of electron and the positive repulsive particles of positron. As the net negative charge of the electron is carried by at least two particles, its net negative charge can only be guaranteed if the total number of the particles making it up is an even integer, the half of which is an odd number. To satisfy this condition, the electron must have at least six building blocks. Out of the infinite number of possible compositions, the assumption that the system is made up of the lowest possible number of building blocks, i.e., six particles, is supported by the fact that the octahedral structure which the particles can form allows for the formation of a stable 3D-structure resistant to forces and rotations.

The conclusion drawn from the above description provides the basis for the TFPF, according to which the smallest, indivisible particles of matter are smaller than the elementary particles, which have been thought to be the smallest. In order to differentiate these particles from the elementary particles, I am going to refer to them as fundamental particles, as "unitrons" (u). Unitrons are the smallest units of matter with invariable properties and they are the building blocks of the elementary particles.

The composition of the elementary particles composed of six unitrons is the following:

$$
\begin{aligned}
& e^{-}=4 u^{-}+2 u^{+}, \\
& e^{+}=2 u^{-}+4 u^{+}, \\
& \gamma^{0}=3 u^{-}+3 u^{+} .
\end{aligned}
$$

The absolute value of the electric charge of the oppositely charged unitrons is equal; this value is half of the net charge of an electron (5). The mass of a unitron is one-sixth of the net mass of an electron (6):

$$
\left|\boldsymbol{q}_{u^{-}}\right|=\left|\boldsymbol{q}_{u^{+}}\right|=\frac{\left|\boldsymbol{q}_{e^{-}}\right|}{2},
$$




$$
\boldsymbol{m}_{u^{-}}=\boldsymbol{m}_{u^{+}}=\frac{\boldsymbol{m}_{e^{-}}}{6} .
$$

According to the TFPF, the attractive and repulsive electric force between unitrons is the only fundamental force. The magnitude of the force is calculated by Coulomb's law. The structure of the elementary particles and the spatial arrangement of their six unitrons can be determined based on the Coulomb forces (Figure 1).

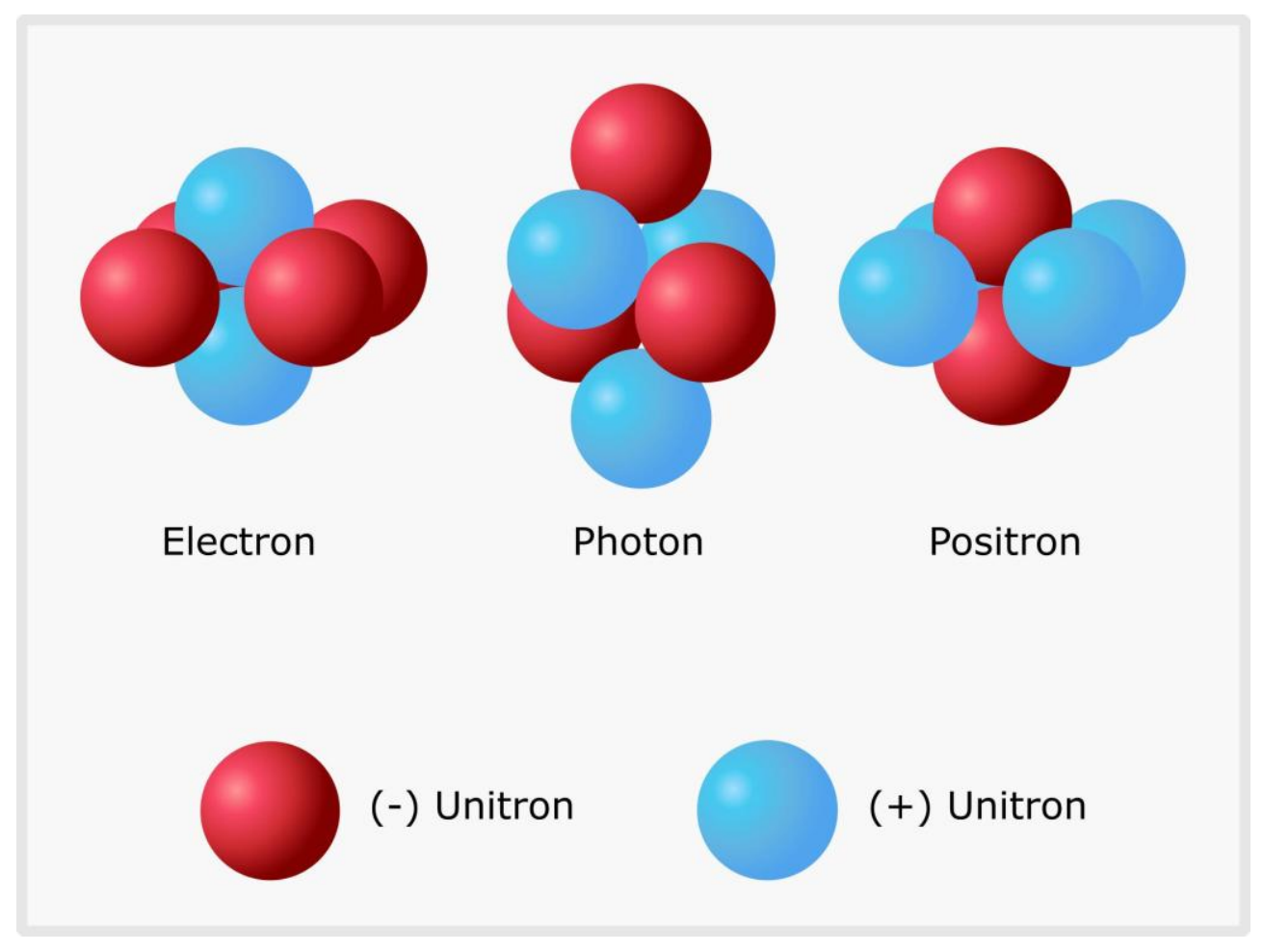

Figure 1. Composition and structure of electron, photon, and positron: The positively and negatively charged unitrons of the photon are located on the vertices of two equilateral triangles of the same size. The planes of triangles are perpendicular and they are embedded into each other along their altitude lines up to their centroids. The frame of electron and positron is a flattened octahedron.

The Universe contains an equal number of positive $\left(\mathrm{u}^{+}\right)$and negative $\left(\mathrm{u}^{-}\right)$unitrons. The mass, spatial extension and electric charge of unitrons are the same and invariable. Mass and electric charge of unitrons were calculated from the measured physical constants of the electron. Their radius was estimated from physical constants of proton presuming a 0.7 packaging density of proton. (Table 1) Unitrons are presumably identical in size and spherical in shape.

Table 1. Invariable Constants of Fundamental Particles.

\begin{tabular}{cccc}
\hline & $\begin{array}{c}\text { Mass } \\
(\mathrm{kg})\end{array}$ & $\begin{array}{c}\text { Electric Charge } \\
(\mathrm{C})\end{array}$ & $\begin{array}{c}\text { Radius } \\
(\mathrm{am})\end{array}$ \\
\hline$(+)$ Unitron $\left(\mathrm{u}^{+}\right)$ & $1.52 \mathrm{E}-31$ & $8.01 \mathrm{E}-20$ & 34.9 \\
$(-)$ Unitron $\left(\mathrm{u}^{-}\right)$ & $1.52 \mathrm{E}-31$ & $-8.01 \mathrm{E}-20$ & 34.9 \\
\hline
\end{tabular}

${ }^{1} 1 \mathrm{am}=1 \mathrm{E}-18 \mathrm{~m}$.

The spontaneous processes of atomic nuclei ( $\left(\beta^{-}\right.$decay, $\beta^{+}$decay, gamma radiation, electron capture); the transformations of protons and neutrons $\left(\mathrm{n}^{0} \rightarrow \mathrm{p}^{+}+\mathrm{e}^{-}\right)$; and the equal charge of the proton and the positron $\left(\mathrm{q}_{\left(\mathrm{e}^{+}\right)}{ }^{+}\right.$ $\left.=\mathrm{q}_{(\mathrm{p})}{ }^{+}\right)$suggest that the electron, the positron, and the photon form integral parts of atomic nuclei. Thus, it can be logically deduced that the building blocks of atomic nuclei are the same as those of the electron, the positron, 
and the photon. According to the extension and generalization of this deduction, any manifestation of the material world is made up of differently structured sets of positively and negatively charged unitrons bound together by fundamental forces. The composition of elementary particles and atomic nuclei can be determined from their mass and net charge (Table 2). The elementary particles and atomic nuclei are composed of unitrons bound together by Coulomb forces. The sum of the fundamental forces holding together the elementary particles may correspond to the strong nuclear force described in modern physics [4].

Table 2. Properties of Elementary Particles

\begin{tabular}{|c|c|c|c|c|c|c|c|c|}
\hline & \multicolumn{2}{|c|}{ Composition } & \multicolumn{2}{|c|}{ Composition } & \multirow{2}{*}{$\begin{array}{c}\text { Extensions } \\
(\mathbf{a m})^{1} \\
\end{array}$} & \multicolumn{2}{|c|}{ Electric charge $(\mathrm{C})$} & \multirow[b]{2}{*}{ net } \\
\hline & $\mathbf{u}^{+}$ & $\mathbf{u}^{-}$ & $\gamma^{0}$ & $\mathrm{e}^{+}$ & & $(+)$ & $(-)$ & \\
\hline Electron $\left(\mathbf{e}^{-}\right)$ & 2 & 4 & - & - & $191 \times 140$ & $1.60 \mathrm{E}-19$ & $-3.20 \mathrm{E}-19$ & $-1.60 \mathrm{E}-19$ \\
\hline Positron $\left(\mathbf{e}^{+}\right)$ & 4 & 2 & - & - & $191 \times 140$ & $3.20 \mathrm{E}-19$ & $-1.60 \mathrm{E}-19$ & $1.60 \mathrm{E}-19$ \\
\hline Photon $\left(\gamma^{0}\right)$ & 3 & 3 & - & - & $161 \times 202$ & $2.40 \mathrm{E}-19$ & $-2.40 \mathrm{E}-19$ & 0 \\
\hline $\operatorname{Proton}\left(\mathbf{p}^{+}\right)$ & 5,509 & 5,507 & 1,835 & 1 & $1,750 \times 1,750$ & 4.4132E-16 & $-4.4116 \mathrm{E}-16$ & $1.60 \mathrm{E}-19$ \\
\hline Neutron $\left(\mathbf{n}^{0}\right)$ & 5,514 & 5,514 & 1,838 & 0 & & 4.4172E-16 & $-4.4172 \mathrm{E}-16$ & 0 \\
\hline $\begin{array}{c}\text { Alpha } \\
\text { particle }\left(\alpha^{2+}\right)\end{array}$ & 21,884 & 21,880 & 7,292 & 2 & & $1.7531 \mathrm{E}-15$ & $-1.7528 \mathrm{E}-15$ & $3.20 \mathrm{E}-19$ \\
\hline
\end{tabular}

${ }^{1} 1 \mathrm{am}=1 \mathrm{E}-18 \mathrm{~m}$.

\section{The Fundamental Force}

Unitrons are the smallest, indivisible units of matter; however, the smallest manifested forms of matter are the elementary particles. Unitrons are bound together by Coulomb forces in elementary particles. These fundamental forces not only bound unitrons together but also determine the position of particles in space and time, i.e. their movement. Coulomb's law can only perfectly describe the fundamental forces under static conditions. It can only use to describe the motion of unitrons if the finite propagation speed of the force is taken into account. The only postulate of the TFPF: the propagation speed of the fundamental force is finite and its value is $\mathrm{c}=299,792,458 \mathrm{~m} / \mathrm{s}$. (This value is equal to the speed of light. The speed of the photon $\mathrm{c}$ is the consequence of the finite propagation speed of the fundamental force.) Since the propagation speed of the force is finite, its effect (depending on the distance and the amount of acceleration) is always delayed in comparison to the accelerated fundamental matter. If it were not so, an immediately occurring force would freeze the world into a motionless state.

The Coulomb force between two unitrons only depends on their distance since their charge can only differ in sign as electric charge is an invariable constant. Thus, the force between two unitrons is:

$$
\boldsymbol{F}= \pm \boldsymbol{k}_{\mathrm{F}} \frac{1}{x^{2}}= \pm \boldsymbol{k}_{\mathrm{F}} \frac{1}{x} \frac{1}{x}
$$

where $\mathbf{F}(\mathrm{N})$ is the force, $\mathbf{k}_{\mathrm{F}}=5.77 \times 10^{-29}\left(\mathrm{Nm}^{2}\right)$ is the modified Coulomb constant (the attractive or repulsive force between two unitrons at a distance of $1 \mathrm{~m}$ ) and $\mathbf{x}(\mathrm{m})$ is the distance between the unitrons.

As a consequence of the finite propagation speed of force, a unitron at equilibrium cannot be displaced at a constant speed, since a net force would act on the unitron in the opposite direction without delay. Moving away from the equilibrium position is only possible by means of acceleration, in the case of which the reaction force with a finite propagation speed is delayed in relation to the accelerated unitron. Thus, the accelerated unitron receives only a Coulomb force corresponding to its earlier position. Therefore, the accelerated motion generates a net force. 
The displacement $(\Delta \mathbf{s})$ is not sufficient in itself to calculate the net force occurring as a result of the motion (Figure 2). In addition, the distance (D) between the interacting unitrons are also needed to determine the magnitude of the force. Thus, the net force generated as a result of the displacement $(\Delta \mathbf{s})$ is:

$$
\boldsymbol{F}=\boldsymbol{k}_{\mathrm{F}} \times\left(\left(\frac{1}{\frac{D}{2}-\frac{\Delta s}{2}}\right)^{2}-\left(\frac{1}{\frac{D}{2}+\frac{\Delta \Delta}{2}}\right)^{2}\right)=\left(\boldsymbol{k}_{\mathrm{F}} \times \frac{16 \times \boldsymbol{D}}{\left(D^{2}-\Delta \boldsymbol{s}^{2}\right)^{2}}\right) \times \Delta \boldsymbol{s}=\boldsymbol{f}_{\mathrm{H}} \times \Delta \boldsymbol{s},
$$

where $\mathbf{F}$ is the net force generated as a result of the displacement, $\mathbf{k}_{\mathrm{F}}$ is the modified Coulomb constant, $\mathbf{D}$ is the distance of the unitrons, $\Delta \mathbf{s}$ is the displacement and $\mathrm{f}_{\mathrm{H}}$ is a multiplier factor.

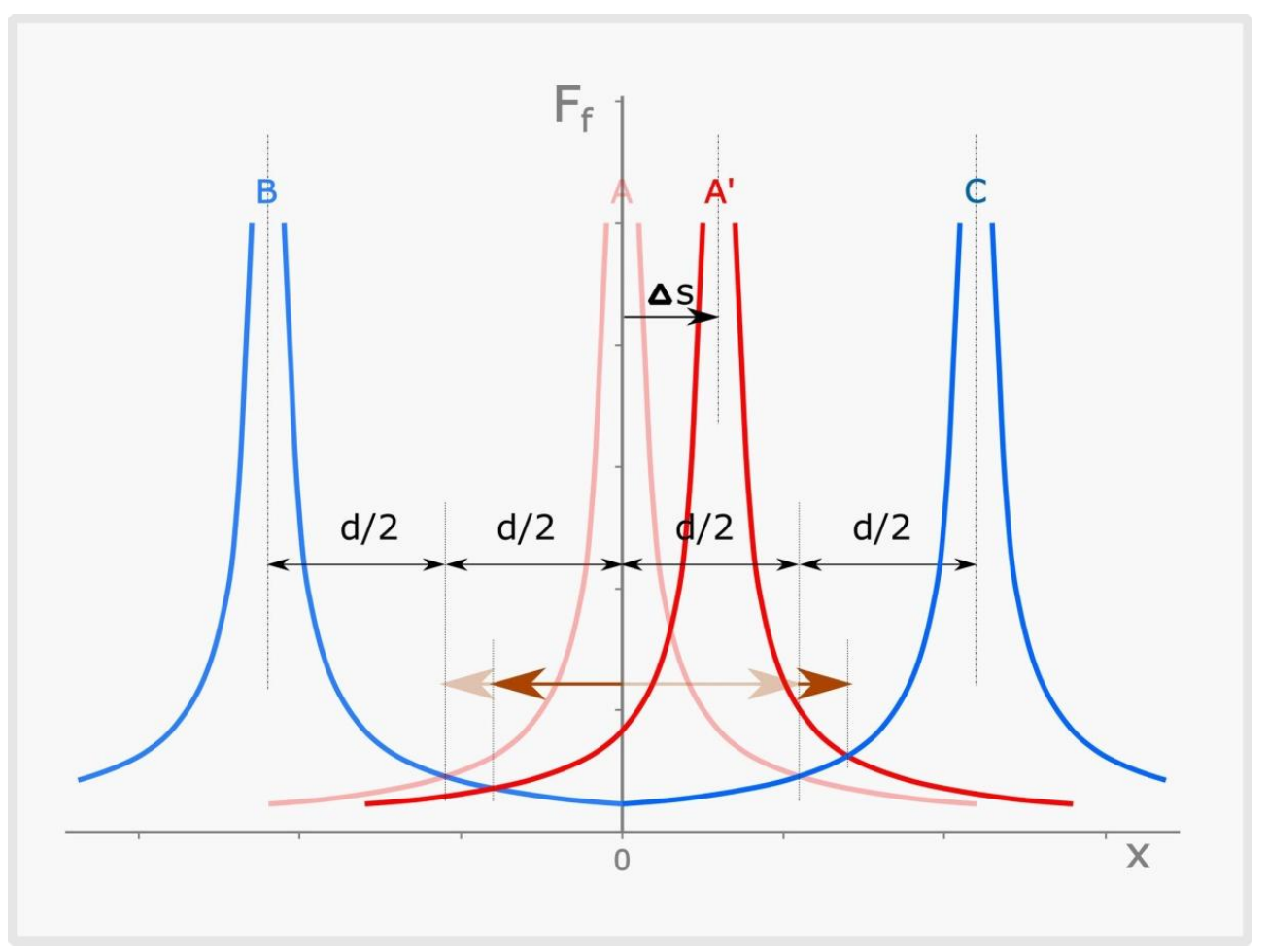

Figure 2. Effect of unitron displacement to the electric field: $\mathrm{X}$ is distance. The displacement of tested unitron from the $\mathrm{A}$ into the $\mathrm{A}^{\prime}$ position $(\Delta \mathrm{s})$ changes the distance $(\mathrm{d})$ and so the forces between unitrons. $\mathrm{F}_{\mathrm{f}}=1 / \mathrm{x}$ referenced to the A, A', B and C positions of unitrons. The plotting expressively demonstrates the alteration of Coulomb forces as the $1 / \mathrm{x}$ values squared at the intersection point of appropriate lines gives the force between the two unitrons.

Important relationships can be inferred based on the simplified product form of the equation. If the displacement of the unitron is much smaller than the distance between the two unitrons ( $\mathbf{D} \gg \Delta \mathbf{s}$ ), the multiplier becomes nearly constant $\left(\mathbf{f}_{\mathrm{H}} \approx\right.$ const). Therefore, the net force generated by the motion is in direct proportion to the displacement from the equilibrium position. Thus, the equation corresponds to Hooke's law; consequently, the force field of the unitrons acts as a spring. However, if two unitrons are at a near-collision distance, the multiplier $\mathbf{f}_{\mathrm{H}}$ is not a constant any longer and as the particles move closer and closer, the generated forces become greater and greater, preventing a collision. For this reason, it can be presumed that at a fundamental level neither the collision of atoms nor that of macroscopic objects involves direct contact. Since displacement can only be realized through acceleration, the force generated by displacement, except for the collision region, is also in proportion to acceleration. Thus, Newton's second law applies. Moreover, upon further consideration, it can be established that each of Newton's laws describing macroscopic objects is of fundamental origin.

In a set of unitrons composing macroscopic objects at dynamic equilibrium, the fundamental forces dynamically balance each other. Considering the whole object, the resultant force is zero, i.e., the object moves in a straight line at a constant velocity not generating force; or, indistinguishable, is at rest. If a force is exerted on the object, each of its unitrons is accelerated, the magnitude of which depends on the number of the unitrons 
making up the object, i.e., it's mass. The force causes the unitrons to move away from equilibrium. Returning to equilibrium generates a reaction of equal magnitude but opposite direction at the speed of light. The mechanical forces displace the unitrons of the object from equilibrium to such a small extent that the linear connection between the force and the acceleration is always realized. Not only Newton's laws are of fundamental origin, but also the macroscopically observable inertia and the inertial forces (the centrifugal force and the Coriolis force) can be explained with the existence of fundamental particles and fundamental forces. This strongly supports the TFPF, i.e., the interaction regulating the Universe is force-based as opposed to the energy-based theory of modern physics.

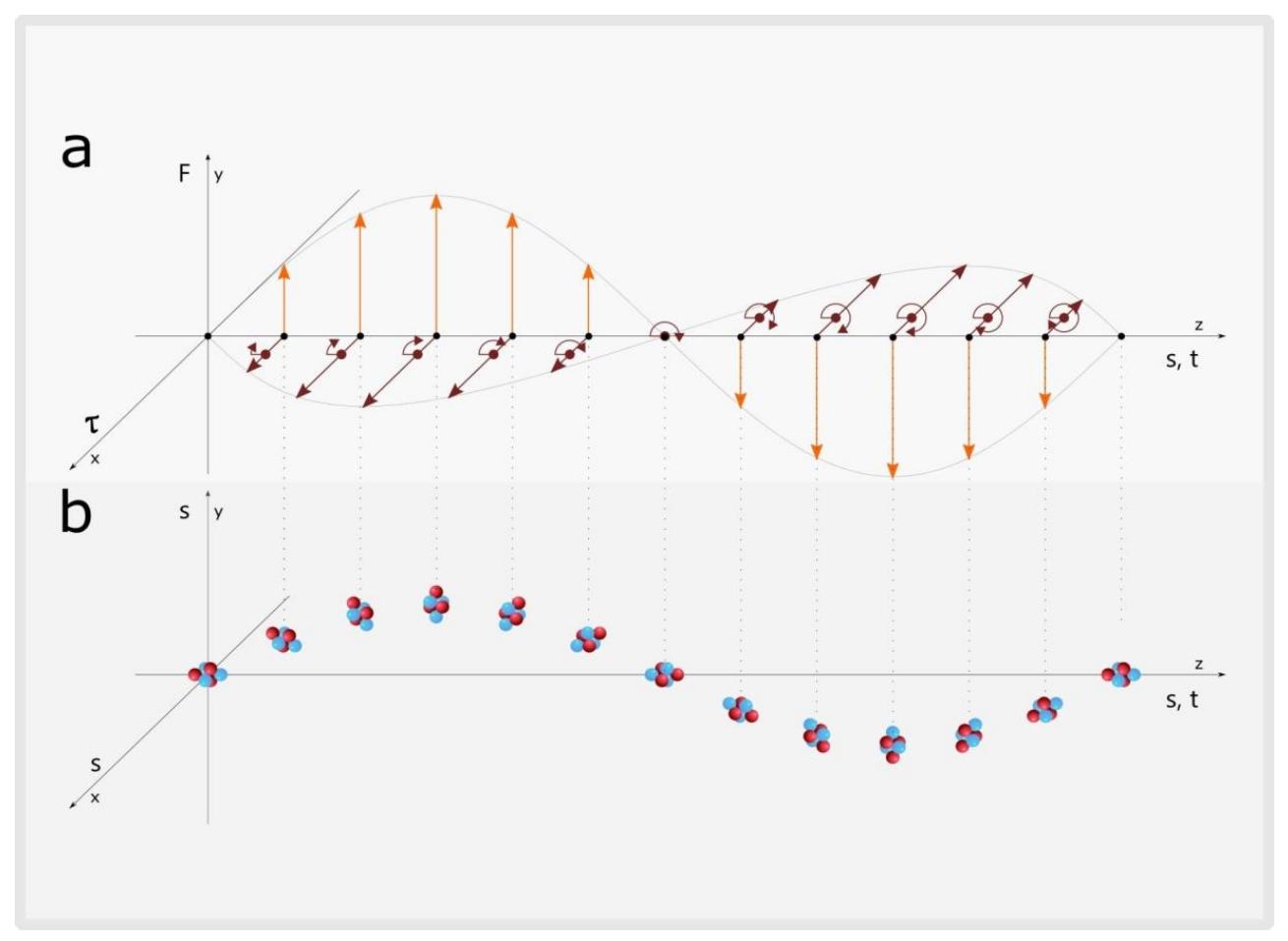

Figure 3. Schematic demonstration of electromagnetic radiation: (a) Oscillation of electric force (F) in ydirection and magnetic force (torque) $(\tau)$ in $\mathrm{x}$-direction as a function of distance $(\mathrm{s})$ and/or time $(\mathrm{t})$. The forces are generated by the spinning photon particle that moves in z-direction; (b) Sinusoidal trajectory of spinning photon particle in the yz-plane.

\section{Electromagnetic Radiation}

The interpretation of electromagnetic radiation is essential for the description of the world at the deepest level. The present model is fundamentally different from modern physics' interpretation of electromagnetic radiation [5]. According to the TFPF, the carrier of electromagnetic radiation is the photon moving at a speed of $299,792,458 \mathrm{~m} / \mathrm{s}$, which is a composite particle spinning around an axis passing through its centre of mass perpendicular to the direction of its motion. Electromagnetic radiation can be made up of single photons, but, especially in lower energy ranges, the occurrence of several photons moving synchronously with each other along the same trajectory is common. The net charge of the photon is zero; thus, it can generate the same amount of attractive and repulsive electric force. This, however, does not mean that it cannot interact with other elementary particles. This is made possible by its rotation and the eccentric distribution of its charges. Photon is a perfect dipole and has an electric dipole moment of approximately $3.85 \times 10^{-38} \mathrm{Cm}$. During its motion, the spinning photon generates a sinusoidal electric field propagating at the speed of light in its plane of rotation and a sinusoidal magnetic field in the direction of its axis of rotation, perpendicular to the electric field (Figure 3).

\subsection{The Electric Force Generated by Spinning Photons}


A photon moving in the $\mathrm{z}$-direction at a velocity c can be considered a dipole rotating at a constant angular velocity in the yz-plane around an x-axis. The equations describing the motion of the positive and negative poles of the photon only differ in a $\pi$ phase shift:

$$
\begin{array}{clrl}
x(t)=0 & y(t)=r \sin (\omega t) & z(t)=r \cos (\omega t)+c t, \\
a_{x}(t)=0 & a_{y}(t)=-r \omega^{2} \sin (\omega t) & a_{z}(t)=-r \omega^{2} \cos (\omega t) .
\end{array}
$$

Far from charged particles (e.g. in a vacuum or in outer space), the acceleration of the charges of the photon does not generate force for lack of interactions. Thus, the photon moves in a straight line at a constant velocity. If it enters the electric field with a dielectric constant greater than that of vacuum, the force generated by spinning charges of the photon is compensated as an action-reaction force pairs by the electric field of the medium. The photon continues its motion along a sinusoidal trajectory perpendicular to its rotation axis. The tangential speed, frequency and period remain unchanged. As a result of the unchanged tangential velocity of the photon, the "wavelength" of the trajectory decreases. The force generated by spinning charges of photon increases in proportion to the angular velocity squared. However, the time and distance available for exerting the force are inversely proportional to angular speed; thus, the product of the force and the distance, that is the work increases only linearly with frequency:

$$
\boldsymbol{W}_{e}=h_{e} \times \boldsymbol{f}
$$

where $\mathbf{W}_{\mathrm{e}}$ is the electric work done as a result of the rotation of the photon, $\mathrm{h}_{\mathrm{e}}$ is a part of the Planck constant and $\mathbf{f}$ is the rotational frequency of the photon.

\subsection{The Magnetic Force Generated by Spinning Photons}

Every elementary particle is a composite; thus, they need to be thought of as rigid bodies. Besides the spatial position of a charged elementary particle, the orientation of the charge pairs making up the elementary particles is also important. The force field of composite elementary particles is direction-dependent; thus, it significantly differs from the force field of point charges. A rotating photon cannot be considered point-like in the direction of the $\mathrm{x}$-axis; however, it is a perfect dipole. Although there is no acceleration and displacement along the $\mathrm{x}$-axis so no electric force is generated, other elementary particles are affected not only by the approaching and receding movement but also by the rotation of the charges. The negative and positive charge of the rotating dipole switch places during a half period, then at the end of a full rotation, it returns to its original state at equilibrium. The positive and the negative charge of the dipole represent two Coulomb forces equal in magnitude but opposite in direction and the point of application is not the same. Therefore, they act as a force pair, exerting torque. The torque transmits a rotational force through the charges of the dipole and it also receives it through the charges of other surrounding dipoles. When calculating the torque, it must be taken into account that the place switching of the charges in the dipole does not happen at rest; instead, it occurs while moving along a sinusoidal trajectory at the speed of light. Therefore, the rotational force does work on the force field determined by the frequency and the amplitude of the trajectory of the photon. In other words, the pivot of the lever will be the centre of the region of space the photon is passing through. The length of the lever varies according to the sinusoidal trajectory; its magnitude can be calculated from the period length and the amplitude of the trajectory of the photon. The rotation of the charges of the photon does not only exert a torque but also a force as a result of the difference in the distance occurring because of the diagonal or opposing position of the charges. The combined effect of the torque and the force on other elementary particles is a vortex interaction. This phenomenon can be considered the basis of the fundamental magnetic interaction. According to the present model, there are no magnetic phenomena independent of the fundamental electric force.

The ability of the magnetic rotational forces to do work is quite similar to that of the linear electric forces. The work with which the rotation of a spinning dipole at a given angular velocity can be stopped or a dipole at rest can be accelerated to a given angular velocity requires a force (torque) that is proportional to the square of the angular velocity. However, in order to exert the effect of the torque, the time available is inversely proportional to the angular velocity. As a combined effect of these factors, the ability of a photon to do magnetic work is in direct proportion to the frequency: 


$$
\boldsymbol{W}_{m}=h_{m} \times \boldsymbol{f}
$$

where $\mathbf{W}_{\mathrm{m}}$ is the magnetic work resulting from the rotation of the photon, $\mathrm{h}_{\mathrm{m}}$ is a part of the Planck constant and $\mathbf{f}$ is the rotational frequency of the photon.

\subsection{Electromagnetic Interactions of the Photon}

The electric and magnetic forces perpendicular to each other are generated by the same charges of the dipole and they are created as a result of the rotation of the dipole. Consequently, although they act according to different mechanisms and in different directions, they decrease or increase synchronously; thus, they are completely connected. Therefore, it is justifiable to use the terms of electromagnetic force and interaction.

Visually, the Universe is an electromagnetic field in which elementary particles move and rotate like gyroscopes (tops, rigid rotors) surrounded by electromagnetic shells. These tops generate and receive purely magnetic, i.e., eddying forces in the direction of their axes of rotation, purely electric, i.e., linear forces in their planes of rotation, while both types of forces, i.e., electromagnetic forces in the directions in between.

The electromagnetic energy of the photon is quantized because the ability of a photon to do work changes sinusoidally due to its dipole nature. Photon is able to do work on the electromagnetic field in every one-half of its rotational periods while the electromagnetic field does work on photon in every other half of periods.

Fast spinning photons (photons of high electromagnetic energy) approach charged particles more and the interaction is more intensive than in case of low spinning photons. The interactions and trajectories of photons are highly dependent on their electromagnetic energy.

According to the present model, the photon is a spinning particle with zero net electric charges moving at a speed $\mathrm{c}$ and having a kinetic energy of $\boldsymbol{E}_{k}=\frac{1}{2} \boldsymbol{m} c^{2}(255.5 \mathrm{keV})$ derived from motion and electromagnetic energy of $\mathbf{E}_{\mathrm{em}}=\mathrm{h} \times \mathbf{f}$ derived from the rotation. The kinetic energy can be calculated but the ability of the photon as a whole to do work is zero. To transfer its kinetic energy originated from its motion, a collision should occur. However, the spinning gyroscope nature of photon averts the collision. The electromagnetic energy of photon originated from spinning should reach a limit for collision. In order for the collision of a photon to occur, both its linear motion and its rotation must be stopped. Therefore, the collision of a photon requires twice as much work as is needed to stop its linear motion as the rotation required to generate the decelerating force must also be slowed down:

$$
\Delta \boldsymbol{E}=\boldsymbol{W}_{e}+\boldsymbol{W}_{m}=\left(h_{e}+h_{m}\right) \times \boldsymbol{f}=h \times \boldsymbol{f}=2 \times \frac{1}{2} \boldsymbol{m}_{\gamma} c^{2}=\boldsymbol{m}_{\gamma} c^{2} \quad(510 \mathrm{keV}),
$$

where $\Delta \mathbf{E}$ is the change in energy, $\mathbf{W}_{\mathrm{e}}$ is the electric work, $\mathbf{W}_{\mathrm{m}}$ is the magnetic work, $\mathrm{h}$ is the Planck constant, i.e., the electromagnetic energy of the photon moving at speed $\mathrm{c}$ and spinning with a frequency of $1 \mathrm{~Hz}, \mathbf{m}_{\gamma}$ is the mass of the photon, $\mathbf{f}$ is its frequency and $\mathrm{c}$ is its speed.

The process in which the photon stops and ceases to rotate can be considered a perfectly inelastic collision occurring in one half of the rotational period. The electromagnetic field does not return the work done by the photon in the absence of the second half of the rotational period of the photon; instead, the work $\left(\mathbf{E}_{\mathrm{b}}=\mathbf{m}_{\gamma} \mathrm{c}^{2}\right)$ is transformed into bonding energy $\left(\mathbf{E}_{\mathrm{b}}\right)$. If the reverse process occurs, photon regains its electromagnetic energy from the bonding energy as it is converted by the work done on the photon $\left(\mathbf{E}_{\mathrm{b}}=\mathrm{h} \times \mathbf{f}\right)$.

According to the TFPF, the famous Einsteinian formula $\mathbf{E}=\mathbf{m c}^{2}$ is a real relationship but it is not about the kinetic energy of the photon as it is only half of this value. The formula does in no way describe an equivalence of matter and energy. The value $\mathbf{E}=\mathbf{m c}^{2}$ must be interpreted as the bonding energy of the photon.

In the case of "annihilation" radiation, the collision can be considered perfectly inelastic as photon loses both its kinetic and electromagnetic energy. Except "annihilation" radiation photon never loses its kinetic energy, i.e. its linear velocity, only its electromagnetic energy can change. Interactions of photon can be elastic or also inelastic from an electromagnetic point of view; accordingly, photons can retain or lose its electromagnetic energy in interactions. An interaction of a photon can be perfectly elastic (e.g. Thomson scattering) or partly elastic (Compton scattering) or perfectly inelastic (e.g. interference or photoelectric effect). However, photon is never annihilated, it is not transformed into energy and it is not generated from energy. Likewise, the emission and radiation are not the birth of a new photon and absorption and extinction are not the annihilation of photons. These events are only changing the electromagnetic energy of photons. 
Consequently, the "absorbed" photons "live on" after their "absorption" and they take part in further interactions involving the gain or loss of energy. Their electromagnetic energy levels are spectrally adapted to the thermal energy levels of the atoms in their surroundings. We can call them "ambient" photons. Electromagnetic radiation is usually distinguished by the energy of photons. The energy of the ambient photons covers the whole electromagnetic spectrum. The word "ambient" does not refer to energy but to function. Ambient photons constitute a pool of individual photons after absorption and before emission.

We can say we are living in an ocean of ambient photons with an electromagnetic energy level describable by the spectrum of black-body radiation, the maximum of which is the same as the temperature of the surroundings. This value is approximately $300 \mathrm{~K}$ on the Earth, $6000 \mathrm{~K}$ in the Sun's corona. The $2.725 \mathrm{~K}$ cosmic background radiation [6] can be explained by the existence of cosmic ambient photons. The explanation of redshift [7] has also differed from the excepted theory. The reason for redshift is the decrease in the electromagnetic energy of photons in the course of its motion. This can be explained by the inelastic electromagnetic interaction of the photon, i.e., an interaction during which the work done by the photon is not returned. Cosmic ambient photons can be suspected of causing such interactions. This kind of photon-photon interaction can mean an unimaginably small loss of energy but its cumulative effect becomes detectable due to the extremely large distance. Assuming that ambient photons which cause cosmic background radiation fill the Universe uniformly, redshift is proportional to the distance of the light source.

\section{Atomic, Subatomic World}

\subsection{Neutron}

According to TFPF, the material world is infinite in space and time. As a consequence, one-way matter transformation processes cannot exist, as this would lead to the accumulation of certain forms of matter. Photons are present in several nuclear reactions but always on the product side except the pair forming. These considerations led to the assumption of the "Solidified Light" (SL) model. The idea is that the photon is not only the carrier of electromagnetic radiation but also a general building block of atomic nuclei. This is only possible if the photon is involved in nucleosynthesis as well. It is assumed that inside certain stars, where forces of sufficient magnitude are possible, neutral photons may condense into likewise neutral neutrons. This process could ensure the dynamic equilibrium between the highly mobile photons and the charged particles possessing limited mobility. The correctness of the SL model can be confirmed or refuted by getting to know the structure of either the neutron or the proton.

It is a purely speculative assumption that neutrons are composed of concentric layers, spherical shells of photons orientating outwards with their negative poles. Of course, the construction from photons is only formal since photons and sets of photons are held together by the same fundamental force. Photons are elastic particles, which can be compressed by $20 \%$. Thus, it can be presumed that a neutron is made up of photons compressed to different degrees. Increased tension and increased surface tension due to geometric reasons can be assumed in the neutron. The neutron generates electromagnetic forces during its rotation, which can cause the elements (especially the ones in asymmetric positions) to be displaced. As a result, the new arrangement changes the direction and magnitude of the electromagnetic forces, which causes further displacements. Presumably, the series of rearrangements means minimal displacements and it could be a process occurring through several variations, which explains the stochastic nature of decay of neutron well. Eventually, the process ends with the ejection of an electron and forming a positron at the point farthest from the axis of rotation of the neutron (Figure 4). In order to interpret beta decay, and, more generally, the phenomenon called weak interaction by modern physics [4], no extra force needs to be assumed besides the fundamental force described by Coulomb's law and the electromagnetic force derived from that.

\subsection{Electron}

Electron, similarly to every other elementary particle, is an electric multipole. If the negative unitrons are formally combined into pairs, the positive and the negative charges get in the same plane and form a rhombus. Therefore, the electron can be considered a quadrupole, even though not a regular one. A characteristic of a quadrupole is that after half a turn it returns to the same position of charges as its original position, as opposed to a dipole, which needs to complete a full turn. During its rotation, the quadrupole generates and receives perpendicular electric and magnetic fields just like the dipole described in the case of the photon. The electron 
can also be considered an electromagnetic top. The force field generated by the electron is the superposition of the Coulomb forces of the two "surplus" negative unitrons and the sinusoidal electric and magnetic forces created by the rotation.

\subsection{Proton}

It can be assumed without knowing its structure that a proton composed of 11,016 unitrons is a spinning electric multipole (Figure 4). According to the SL model, the proton created by the decay of a neutron is made up of 1,835 photons and 1 single positron on the outer surface of the proton. The composition of the proton hardly differs from that of the neutron, but the single positron, which makes them different, means a great change both in the density and the electromagnetic characteristics of the proton. The eccentric position of the net positive charge of the positron causes a significant asymmetry in the multipole structure of the proton.

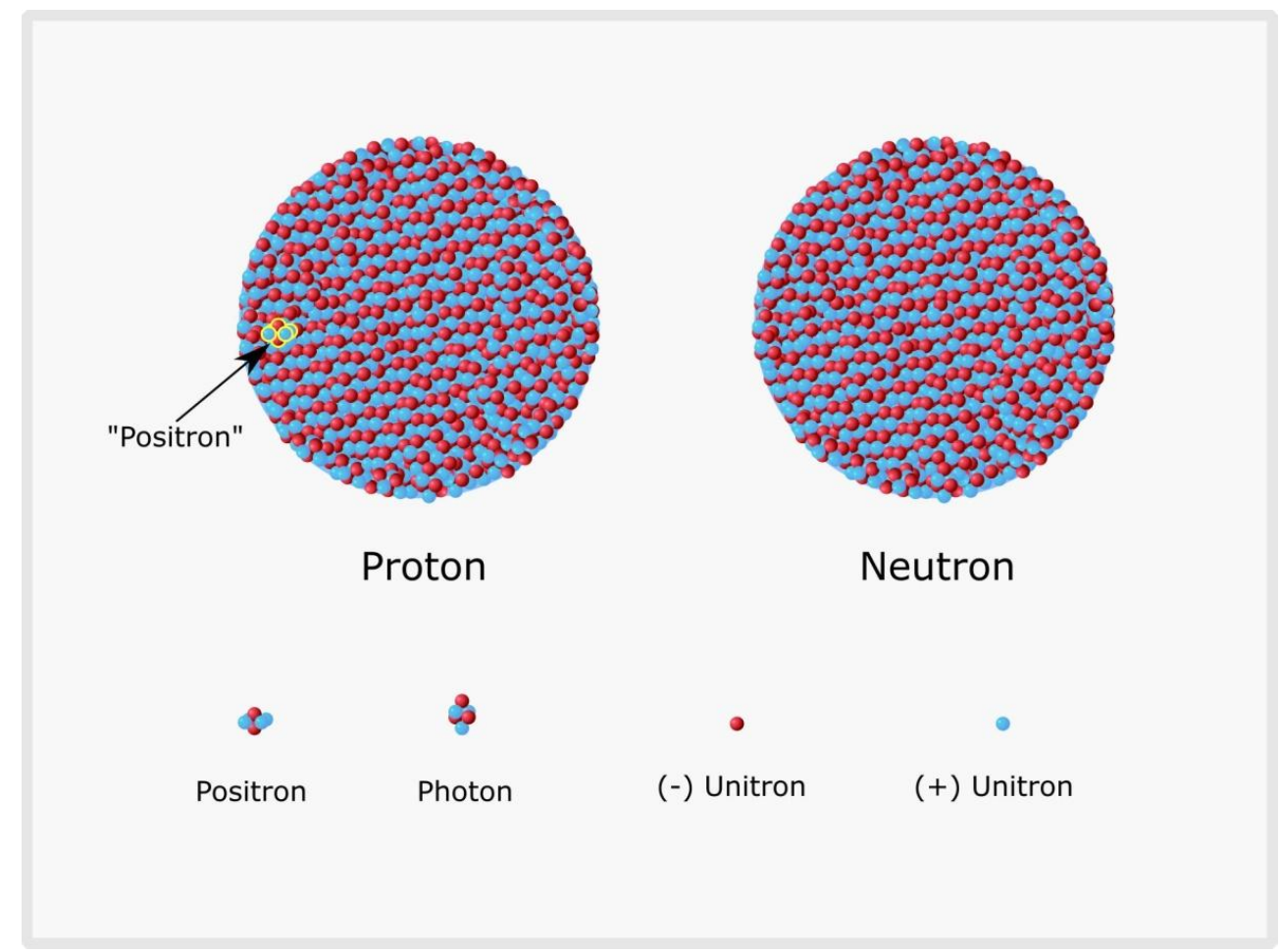

Figure 4. Illustration of proton and neutron: Real relative extensions, untrue structure.

\subsection{Hydrogen Atom}

It can be presumed that the axes of rotation of the proton and the electron in a hydrogen atom are parallel to each other and the two electromagnetic tops rotate and oscillate synchronously. The motion of particles is determined by the combined effect of their own inertia and the external forces acting on them. Focusing on the electron, it can be said that as a result of its own inertia, the electron would, in theory, move along a straight line at a constant velocity. However, the net positive charge of the proton causes the electron to move in a circular orbit, the magnetic force of the proton results in the rotation of the electron, while the electric force of the proton makes the electron oscillate. The combination of the oscillation and circular motion of the electron means that the electron moves along a sinusoidally modified circular trajectory. To sum up, the motion of the proton and the electron in the hydrogen atom is synchronous and they form parts of the same system.

The oscillation combined with the rotation of the electron-proton system (i.e., a system made up of a rotating quadrupole and a rotating multipole) is a rather complex process. Stable oscillation, i.e., resonance can only occur if the electromagnetic forces created during the rotation and oscillation of one of the particles are perfectly compensated by those forces generated during the rotation and oscillation of the other particle. Since the mass of the proton is greater than that of the electron by several orders of magnitude, the role of the proton is the determining factor in creating equilibrium. For resonance to occur, the electron is forced to move along its 
orbit at the angular velocity and amplitude determined by the electromagnetic forces due to the structure of the proton. As a result of the multipole structure of the proton, the electron can fulfill the condition for steady-state oscillation by moving on several different orbits of precisely defined parameters. These are orbits of different discrete energy levels; thus, the different electromagnetic forces are exchanged between the proton and the electron along distances of different length. In its ground state, the electron moves on the orbit that requires the least amount of work. In this case, the ability of the electron to do work is zero. The electron can only reach higher-energy states, i.e., excited states if an external electromagnetic force acts on it. Since every possible energy level of the electron is discrete, only the work done by those external forces can be realized that are able to bridge the energy difference between the possible electron orbits.

This process can be well detected in the case of the interaction between a hydrogen atom and a photon. A photon approaching the hydrogen atom, an electromagnetic interaction can occur between the electron and the photon, which can be either elastic or inelastic. In the case of an elastic interaction, the electron returns the work done on it to the photon. Thus, the electromagnetic energy of the photon does not change during the interaction and as a result, no change is spectroscopically detectable. An inelastic interaction occurs when the amount of the electromagnetic energy of the photon is the same as the work needed for the electron to jump to a higher energy level. In this case, the electron does not return the work done on it because this work is transformed into potential energy. The photon, which loses its electromagnetic energy but preserves its kinetic energy, leaves as an ambient photon and it will disappear from the spectrum. The process can also take place in reverse when an excited atom, i.e., its higher-energy electron increases the energy of an ambient photon by doing work on it, which is also spectroscopically detectable. In this case, the electron falls back to a lower energy level corresponding to the energy dissipated by the work done.

The interaction between the photon and the electron does not affect the electrostatic interaction between the proton and the electron. Thus, as opposed to the currently accepted theory, it does not change the average distance between the proton and the electron in a hydrogen atom. The average size of an excited hydrogen atom is the same as that of a hydrogen atom in the ground state. The most important difference between what is currently accepted and the TFPF is that according to the latter the properties of atoms cannot be derived from those of the electrons; instead, they are principally determined by atomic nuclei. The motion of an excited electron around the proton and its excited states are not determined by the quantum numbers, but by the multipole structure of the proton, i.e., the spatial distribution of the unitrons which compose it. The continuous spectrum of synchrotron radiation proves that "quantum numbers" do not stem from the intrinsic properties of the electron; instead, they are encoded into the spatial distribution of the unitrons composing protons and atomic nuclei. The answer to the Rydberg formula and every physical and chemical property of a hydrogen atom is encoded into the structure of the proton, even if the electron has an indisputably important role in implementation. The road to developing a new, realistic atomic model leads through the exploration of the structure of the proton.

\subsection{Atomic Nuclei}

The structure of complex atoms, which contain more than one proton (positron), and the orbits of the electrons around them - similarly to hydrogen atoms - are determined by the structure of the atomic nucleus. However, it is complemented by the partly electromagnetic, partly electrostatic effects of the electrons on the nucleus and on each other.

According to TFPF, the expected structure of complex atomic nuclei is significantly different from the currently accepted configuration. Complex nuclei are not composed of protons and neutrons but are created from protons and neutrons. Consequently, the cause of the mass defect is not the transformation of matter into energy, but the fact that during nucleosynthesis the photons that are "superfluous" as far as the optimal geometric structure of the fusing nuclei is concerned are ejected from the atomic nucleus. The released bonding energy is carried by the products in the form of kinetic and electromagnetic energy. It can be assumed that stable complex nuclei are created from the fusion of presumably more loosely structured protons and more compact neutrons present at a nearly optimal ratio. The numbers of emitted photons can be calculated from the quotient of the mass defect and the mass of a single photon. The formula $\mathrm{E}=\mathrm{mc}^{2}$ can be used in calculations as the average bonding energy of a photons, but it cannot be used as a general formula.

It is true both for protons and complex nuclei that their structure is of crucial importance in determining atomic properties. Atoms can be sorted into a periodic table based on their properties, but nonetheless, each atom 
has unique characteristics that are different from those of any other atom. This is difficult to explain with the currently accepted theory, but it corresponds very well to the TFPF model, according to which thousands of unitrons, with their diverse spatial arrangement, allow for the formation of electric multipole structures with unique properties, i.e., individual atomic nuclei. The properties of the atomic nucleus are reflected in the orbits of the surrounding electrons and, ultimately, in the distinctive properties of the atom. Much more refined and more realistic image of atoms can be created than the currently accepted system of the positive nucleus and negative electrons.

The orbits of the electrons around the atomic nucleus are formed by the interactions of the nucleus and other electrons. Since both atomic nuclei and electrons can be described as electromagnetic gyroscopes, it may be possible to characterize atoms with the Euler angles characteristic of the rotation of rigid bodies instead of the currently accepted magic quantum numbers. The atomic nucleus does not only act on the electrons surrounding it, but it also enters into electrostatic and electromagnetic interactions with the electrons and nuclei of adjacent atoms. Thus, in contrast to the currently accepted electron-based explanation, atomic nuclei play a crucial role in the formation of chemical bonds.

\section{Gravity}

Because of the formal similarity between the equations describing gravity and the electrostatic interaction, it has long been suspected that there is a relationship between the two phenomena. The ratio between the electrostatic and gravitational forces is $3.75 \times 10^{43}$, assuming the same distance and unitron number. According to the TFPF gravity can be explained with a special manifestation of the fundamental forces between the unitrons. Gravity is derived from the motion of elementary particles and from the different nature of electric attraction and repulsion.

The elementary particles and atoms can be described as electromagnetic gyroscopes. Consequently, macroscopic objects can be considered to be sets of multipoles spinning around their axes. Multipoles, on the other hand, can be treated as sets of elementary electric dipoles. Since the electric forces generated by the dipoles in their plane of rotation are additive, a sinusoidally alternating, pulsing electric field is formed around all macroscopic objects and propagated in all directions of space at the speed of light. Thus, elementary spinning dipoles of objects exert sinusoidally altering attractive and repulsive forces on every unitron of all objects. (The magnetic forces do not play a role in the phenomenon of gravity.) The time average of electric forces generated by elementary dipoles is zero but the sinusoidally altering attractive and repulsive forces will pull and push the unitrons. However, the displacements of unitrons will be asymmetrical. The attractive force exerting on approaching unitron will increase during the displacement as the distance will decrease. Conversely, the repulsive force exerting on unitron moving away will decrease during the displacement. The difference between the increasingly attractive and decreasingly repulsive forces during a period of pulsing electric field gives a net elementary attractive force. The unitrons cover a greater distance during the attraction phase than in the opposite direction during the repulsion phase. Thus, the equilibrium points of the unitrons are closer to the forcegenerating object at the end of the attraction-repulsion cycle than they were before the cycle. The sum of the net elementary attractive forces gives gravitational force. Gravity is quantized, as it is a sum of elementary forces. The gravity is the common effects of the self-amplifying nature of attraction and the self-weakening nature of repulsion on one hand and the movement and gyroscope-like nature of elementary particles and atoms on the other.

As each unitron in each object interacts with each unitron in the other object, the gravitational force is proportional to the product of the number of the unitrons involved, i.e., the product of the masses of the objects, and the reciprocal of the square of the distance between the objects. The factor of proportionality is a constant resulting from the general structure and fundamental motion of matter.

Although gravity can be calculated from mass, i.e., from the number of unitrons, the cause of gravity does not lie in mass but in the fundamental motion of electrically charged particles. In the centers of supernovae, where the atoms are crushed by gravity, the motion of the unitrons nearly stops. Since gravity is a consequence of the motion of the fundamental particles, the generation and acceptation of gravity are eliminated as the motion of fundamental particles ceases. The core of the supernova becomes a gigantic metastable set of unitrons which then explodes. This disintegration is not only important for the formation of heavier atomic mass chemical elements, but also for the spreading of material condensed by gravity - ensuring aggregation of material not to be a one-way process. 


\section{Discussion}

The basic principles of the Universe described by the TFPF are incredibly simple. The Universe consists of two types of electrically charged fundamental particles and the attractive and repulsive electric forces determining their position in space and time. The electric charge as a macroscopic term could be confusing in the fundamental level. The electric charges can be considered as the organization of substance according to a lefthanded and right-handed symmetry. However, the intrinsic forces of unitrons, the implementation of attraction and repulsion will remain a mystery probably forever. Despite the simplicity of the fundamental system, it can create the diversity of the world by developing from simple structures towards more complex ones. Because of the differences between the basics and the theoretical principles used for the description of the Universe, this model describes electromagnetic radiation, gravity, electromagnetic forces, the atomic and the subatomic world completely different from the theory accepted by modern physics. According to the TFPF, beyond time, space and matter, only the assumption of the existence of the intrinsic force is needed to interpret the Universe. Coulomb's law, Newton's laws, the laws of conservation of mass and electric charge are necessary and sufficient to describe the Universe on the fundamental level. Completing this alternative concept outlined by the TFPF requires a huge amount of work; however, it holds the possibility of getting to know the world more precisely.

\section{References}

[1] Donoghue, J.F., Golowich, E., Holstein, B., Dynamics of the Standard Model, Cambridge University Press, Cambridge (2014).

[2] De Aquino, F. Theory of Everything, arXiv:gr-qc/9910036 (1999).

[3] Charlton M., and Humberston J. W., Positron Physics, Cambridge University Press (2001).

[4] Kane G. L., Modern Elementary Particle Physics, Perseus Books (1987).

[5] Purcell E. M., and Morin D. J., Electricity and Magnetism, Cambridge University Press (2013).

[6] Durrer R., The Cosmic Microwave Background, Cambridge University Press (2008).

[7] Sachs R. K., and Wolfe A. M., Astrophys. J., 147, 73 (1967). 